\title{
FATORES ASSOCIADOS À PROPAGAÇÃO DE INFECÇÕES SEXUALMENTE TRANSMISSÍVEIS ENTRE IDOSOS NO BRASIL: UMA REVISÃO DA LITERATURA
}

\section{FACTORS ASSOCIATED WITH SEXUALLY TRASMITTED INFECTIONS SPREADING AMONG THE ELDERLY IN BRAZIL: A LITERATURE REVIEW}

\section{Laís Borges Sales (SALES, L. B.)}

Acadêmica do Curso de Medicina do Centro Universitário de Anápolis - UniEVANGÉLICA, Anápolis-GO, Brasil. laisborges.sales@gmail.com

Juliana Yasmim Mendonça Leão de Oliveira (OLIVEIRA, J. Y. M. L.)

Acadêmica do Curso de Medicina do Centro Universitário de Anápolis - UniEVANGÉLICA, Anápolis-GO, Brasil. Juliianaleao3@gmail.com

\section{Luma de Oliveira Campanholo (CAMPANHOLO, L. O.)}

Acadêmica do Curso de Medicina do Centro Universitário de Anápolis - UniEVANGÉLICA, Anápolis-GO, Brasil. lumacampanholo@gmail.com

Matheus Henrique Mendes de Oliveira (OLIVEIRA, M. H. M.)

Acadêmico do Curso de Medicina do Centro Universitário de Anápolis - UniEVANGÉLICA, Anápolis-GO, Brasil. matheusmendesmedicina@gmail.com

\section{Rogério Fagundes Vicente (VICENTE, R. F.)}

Acadêmico do Curso de Medicina. Centro Universitário de Anápolis - UniEVANGÉLICA, Anápolis-GO, Brasil. rogeriofv@ hotmail.com

\section{Renata Silva do Prado (PRADO, R. S.)}

Docente do Centro Universitário de Anápolis - UniEVANGÉLICA, Anápolis-GO, Brasil. renata.ufg.prado@gmail.com

Endereço para correspondência:

Rua das Oliveiras, quadra 17, lote 6, n. 97, Jardins Valência, Goiânia-GO. Brasil. CEP: 74885-853. Fone: (62) 99223-0001. E-mail: laisborges.sales@ gmail.com 


\section{RESUMO}

Introdução: A transição demográfica, marcada pelo envelhecimento populacional, tem como consequência o prolongamento no período que diz respeito à atividade sexual da população idosa. Uma das complicações que surgem nesse contexto é o aumento da transmissão de Infecções Sexualmente Transmissíveis (IST) entre os idosos no Brasil. Objetivo: Definir os principais fatores que influenciam na transmissão de IST entre a população idosa no Brasil. Metodologia: Trata-se de uma revisão integrativa da literatura, na qual foram buscados artigos publicados entre 2015 e 2020, nos seguintes bancos de dados: National Library of Medicine and National Institutes of Health (PUBMED), Literatura Latino-Americana e do Caribe em Ciências da Saúde (LILACS), Google Scholar e Scientific Eletronic Library Online (SCIELO). Resultados e discussão: Ao final foram selecionados 25 artigos. Os principais fatores associados à transmissão de IST entre idosos são: a desinformação por parte dessa população e dos profissionais de saúde, os aspectos culturais e a contínua prática sexual insegura. Há a compreensão errônea de que preservativos têm função apenas de contracepção, além de baixo conhecimento sobre a transmissão de IST por parte desse grupo populacional. Ademais, a formação dos profissionais de saúde não abrange de maneira efetiva a sexualidade do idoso, o que corrobora com atitudes conservadoras por parte dos profissionais. Conclusão: Os aspectos cultutais, a desinformação e as práticas sexuais inseguras continuam contribuindo para o aumento na transmissão de IST no país, o que necessita, portanto, de desenvolver políticas públicas que englobem o idoso nas campanhas de prevenção.

Palavras-chave: Saúde do idoso. Sexualidade. Serviços de saúde para idosos. Saúde sexual. Infecções sexualmente transmissíveis.

\section{ABSTRACT}

Introduction: The demographic transition, marked by population aging, has as consequence the extention of the period associated with the sexual activity of the elderly population. One of the complications that arise in this context is the increasing in transmission of Sexually Transmitted Infections (STI) among the elderly in Brazil. Objective: To define the main factors that influence on the transmission of STI among the elderly population in Brazil. Methods: This is an integrative review of the literature based on articles searched between the period of 2015 and 2020. The databases used were National Library of Medicine and National Institutes of Health (PUBMED), Latin American and Caribbean Health Sciences Literature (LILACS), Google Scholar and Scientific Electronic Library Online (SCIELO). Results and discussion: Ultimately, 25 articles were selected. The main factors associated with the transmission of STI among the elderly are the misinformation that this population and health professionals have, cultural aspects and continuous unsafe sexual practices. There is a misconception that condoms have a contraceptive function only, besides little knowledge about STI transmission among this population group. Furthermore, the education and training of health professionals does not eff ectively reach the sexuality of the elderly, which only endorses conservative attitudes by professionals. Conclusion: Cultural aspects, misinformation and unsafe sexual practices continue to contribute to the increase in the transmission of STI in the country, which therefore requires the development of public policies that include the elderly in prevention campaigns.

Keywords: Healthy of the Elderly. Sexuality. Health Services for the Aged. Sexual Health. Sexually transmitted infections. 


\section{INTRODUÇÃO}

A população brasileira, assim como a de diversos países em desenvolvimento, tem vivenciado um processo de transição demográfica, marcada pelo envelhecimento populacional. Essa mudança traz consigo diversos problemas que influenciam diretamente os sistemas de saúde, os quais tiveram que entrar em um processo de adequação a esta nova realidade (SOUSA et al., 2020). Em consonância a isso, a longevidade expressa por indivíduos com mais de 60 anos tem sido inversamente proporcional à obtenção de qualidade de vida, evidenciada pelas fragilidades dos senescentes, pelas doenças crônico-degenerativas, e pela sensação de dependência e inutilidade (MIRANDA; MENDES; SILVA, 2016).

Com os notáveis avanços da medicina e da tecnologia, a população idosa tem conquistado um prolongamento no período que diz respeito à sua atividade sexual. Os idosos se tornaram mais ativos mediante o uso de medicamentos, próteses para disfunção erétil, e ainda a reposição hormonal (SANTOS et al., 2017). Contudo, em paralelo com esses avanços, o número de infecções sexualmente transmissíveis (IST) nessa faixa etária vivencia uma curva crescente de acometimento (LUZ et al., 2015).

Durante muito tempo construiu-se na sociedade a visão de que o idoso é um ser dotado de limitações. Essa construção cultural fez com que surgissem diversos tabus relacionados à possibilidade de se manter uma vida sexual ativa na velhice (NARDELLI et al., 2016). Dessa forma, têm-se cada vez mais banalizado o fato de que os idosos também apresentam riscos no que se refere a infecções sexualmente transmissíveis. Os próprios profissionais de saúde, em consequência de possuírem uma ideia enraizada, são capazes de negligenciar o atendimento aos idosos vulneráveis a esse tipo de situação (ANDRADE; BENITO, 2016).

O fato de os idosos não serem vistos pela população em geral como vulneráveis ao acometimento por IST, acaba por influenciar nas medidas de prevenção que deveriam ser realizadas, tal como o uso de preservativos, que não é frequente entre essa população, sendo inclusive seis vezes menor quando comparado à população jovem (SANTOS et al., 2017). Consequentemente, têm-se percebido mudanças no curso epidemiológico de doenças como a Síndrome da Imunodeficiência Adquirida (AIDS), dado que o número de casos entre idosos continua aumentando significativamente (THEIS; GOUVÊA, 2019). Os profissionais de saúde têm um papel fundamental nesse processo de prevenção e orientação, porém, devem ser isentos de preconceitos, aprimorando-se a fim de não enxergar o idoso apenas como seres em degeneração (EVANGELISTA et al., 2019). 
A política nacional de saúde da pessoa idosa (PNSPI) instituída em 2006 e implementada na saúde pública, tem um papel fundamental no processo de promoção à saúde a esta população, visto que possui como uma de suas diretrizes o provimento de recursos capazes de assegurar qualidade da atenção à saúde da pessoa idosa. Além disso, também se torna muito importante no provimento de informação entre os profissionais da saúde, tendo como função a formação e educação permanente desses profissionais de saúde para lidar com esse púbico (BRASIL, 2006).

Embora seja evidente o atual envelhecimento da população e a questão da vulnerabilidade dos idosos frente às IST, há ainda pouco estudo sobre essa problemática, principalmente no Brasil (ANDRADE et al., 2017). Esse fato corrobora com a demora na adoção de políticas de prevenção, e com a ausência de ações educativas em saúde relacionadas à prevenção de IST em idosos, fazendo com que esse público se torne cada vez mais distante da informação, da testagem e do diagnóstico precoce dessas doenças (FERREIRA et al., 2019).

Desta forma, a escassa literatura a respeito da sexualidade na terceira idade dificulta o acesso à informação à população e permite a continuidade da transmissão de infecções sexuais nessa faixa etária. Logo, em função tanto de aspectos culturais na sociedade quanto de negligência por parte dos profissionais da saúde, o presente estudo faz-se necessário.

Portanto, o objetivo desse trabalho é definir os principais fatores que influenciam na transmissão de Infecções Sexualmente Transmissíveis (IST) entre a população idosa no Brasil, pormenorizando as justificativas e questões associadas a esses fatores.

\section{METODOLOGIA}

O estudo trata-se de uma revisão integrativa da literatura, no qual realizou-se uma coleta de dados a partir de estudos originais, com o propósito de definir os fatores que possuem influência na transmissão de IST entre idosos no Brasil. Foram buscados artigos publicados entre 2015 e 2020 e os bancos de dados utilizados para a pesquisa foram National Library of Medicine and National Institutes of Health (PUBMED), Literatura Latino-Americana e do Caribe em Ciências da Saúde (LILACS), Google Scholar e Scientific Eletronic Library Online (SCIELO). Os seguintes descritores foram utilizados no processo de busca dos artigos, assim como suas combinações tanto em português quanto em inglês com os operadores booleanos AND e OR: saúde do idoso, sexualidade, serviços de saúde para idosos, doenças sexualmente transmissíveis e saúde sexual. A questão norteadora 
da pesquisa foi: quais os principais fatores associados à transmissão de IST em idosos no Brasil?

As buscas e análise dos artigos foram realizadas no período entre agosto e setembro de 2020, e foram definidos critérios de inclusão e exclusão. Os critérios de inclusão utilizados foram a seleção de artigos que relacionam a propagação de infecções sexualmente transmissíveis entre idosos no Brasil e seus diversos fatores condicionantes, com participantes idosos diagnosticados com IST, e artigos que retratam a realidade atual em diversas regiões do país. Os critérios de exclusão utilizados foram: artigos não publicados nos últimos 5 anos, artigos que não tratam de fatores que se associam às IST em idosos e estudos que não foram realizados em regiões brasileiras.

\section{RESULTADOS E DISCUSSÃO}

Com o propóstito de responder à pergunta norteadora foram selecionados artigos publicados nos últimos 5 anos, e ao final foram selecionados 25 artigos. Os resultados foram elencados em três categorias: desinformação por parte dos idosos e dos profissionais de saúde, práticas sexuais inseguras e aspectos culturais. A síntese dos artigos utilizados e os principais resultados encontrados estão dispostos no quadro abaixo.

Quadro 1. Apresentação da síntese de artigos utilizados nesta revisão integrativa.

\begin{tabular}{|c|c|c|c|c|}
\hline Título do artigo & $\begin{array}{l}\text { Autor } \\
\text { principal/ } \\
\text { ANO }\end{array}$ & Tipo de estudo & $\begin{array}{l}\text { Instrumento } \\
\text { utilizado }\end{array}$ & Resultado \\
\hline $\begin{array}{l}\text { Perfil da sexualidade de pessoas } \\
\text { idosas portadoras de SIDA/AIDS } \\
\text { atendidas em um serviço de } \\
\text { saúde do Distrito Federal }\end{array}$ & $\begin{array}{l}\text { ANDRADE, } \\
\text { P.B.S.; } \\
\text { BENITO, } \\
\text { L.A.O. } 2016\end{array}$ & $\begin{array}{l}\text { Pesquisa de } \\
\text { campo } \\
\text { exploratória, de } \\
\text { natureza } \\
\text { descritiva e } \\
\text { quantitativa. }\end{array}$ & $\begin{array}{l}\text { Entrevista: } \\
\text { Escala de } \\
\text { Atitudes e } \\
\text { Conhecimento } \\
\text { sobre } \\
\text { Sexualidade } \\
\text { no } \\
\text { Envelhecimen } \\
\text { to (ASKAS) }\end{array}$ & $\begin{array}{l}\text { Crescimento de casos entre indivíduos } \\
\text { heterossexuais. } 70 \% \text { dos entrevistados } \\
\text { acham que o abandono da atividade } \\
\text { sexual em idosos está ligado diretamente } \\
\text { aos fatores sociais e psicológicos. }\end{array}$ \\
\hline $\begin{array}{l}\text { Sexuality in old age: } \\
\text { knowledge/attitude of nurses of } \\
\text { Family Health Strategy }\end{array}$ & $\begin{array}{l}\text { EVANGELIS } \\
\text { TA, A.R. et } \\
\text { al. } 2018\end{array}$ & $\begin{array}{l}\text { Estudo } \\
\text { transversal, de } \\
\text { natureza } \\
\text { exploratória, } \\
\text { descritiva e } \\
\text { quantitativa. }\end{array}$ & Entrevista & $\begin{array}{l}\text { "Em geral, as mudanças na sexualidade } \\
\text { das pessoas com mais de } 65 \text { anos têm } \\
\text { mais relação com respostas mais lentas } \\
\text { do que com diminuição do interesse por } \\
\text { sexo", } 58,93 \% \text { assinalaram verdadeira, } \\
17,86 \% \text { falsa, e } 23,21 \% \text { não sei. }\end{array}$ \\
\hline $\begin{array}{l}\text { Qualidade de vida, perfil } \\
\text { socioeconômico, conhecimento e } \\
\text { atitude sobre sexualidade de } \\
\text { "pessoas que vivem" com o } \\
\text { Vírus da Imunodeficiência } \\
\text { Humana. }\end{array}$ & $\begin{array}{l}\text { OKUNO, } \\
\text { M.F.P. et al. } \\
2015\end{array}$ & $\begin{array}{l}\text { Estudo } \\
\text { transversal, de } \\
\text { natureza } \\
\text { analítica. }\end{array}$ & Questionário & $\begin{array}{l}\text { Observa-se que os pacientes do sexo } \\
\text { feminino e desempregados foram os que } \\
\text { apresentaram maior conhecimento sobre } \\
\text { sexualidade de idosos, enquanto no } \\
\text { domínio atitudes os pacientes com maior } \\
\text { escolaridade e com idade inferior aos } 60 \\
\text { anos foram os que apresentaram atitudes }\end{array}$ \\
\hline
\end{tabular}




\begin{tabular}{|c|c|c|c|c|}
\hline & & & & mais favoráveis à sexualidade de idosos. \\
\hline $\begin{array}{l}\text { Avaliação da abordagem médica } \\
\text { da sexualidade em idosos com } \\
\text { dor crônica. }\end{array}$ & $\begin{array}{l}\text { CHERPAK, } \\
\text { G.L.; } \\
\text { SANTOS, } \\
\text { F.C. } 2016\end{array}$ & $\begin{array}{l}\text { Estudo } \\
\text { transversal, de } \\
\text { natureza } \\
\text { analítica } \\
\text { descritiva. }\end{array}$ & Questionário & $\begin{array}{l}\text { A maioria dos médicos informou não } \\
\text { abordar o assunto de sexualidade. Dentre } \\
\text { os que falavam sobre o assunto, } 29,63 \% \\
\text { haviam sido treinados, enquanto entre os } \\
\text { que não mencionavam o assunto, } 21,27 \% \\
\text { foram treinados. }\end{array}$ \\
\hline $\begin{array}{l}\text { Conhecimento sobre síndrome } \\
\text { da imunodeficiência humana de } \\
\text { idosos de uma unidade de } \\
\text { atenção ao idoso. }\end{array}$ & $\begin{array}{l}\text { NARDELLI, } \\
\text { G.G. et al. } \\
2016\end{array}$ & $\begin{array}{l}\text { Estudo } \\
\text { transversal, de } \\
\text { natureza } \\
\text { descritiva e } \\
\text { quantitativa. }\end{array}$ & Questionário & $\begin{array}{l}\text { No que se refere ao uso de preservativo, } \\
\text { um total de } 298 \text { idosas }(88,2 \%) \text {, disseram } \\
\text { que não fazem uso de preservativo e } 62 \\
\text { idosos }(52,1 \%) \text { também disseram não } \\
\text { utilizar preservativo, apesar de } 88,1 \% \text { e } \\
89,4 \% \text { considerando os idosos de ambos } \\
\text { os sexos terem relatado respectivamente } \\
\text { que, devem se preocupar com adquirir o } \\
\text { HIV/AIDS e que a camisinha previne } \\
\text { essa transmissão. }\end{array}$ \\
\hline $\begin{array}{l}\text { Vulnerabilidade a infecções } \\
\text { sexualmente transmissíveis em } \\
\text { idosos usuários de um centro de } \\
\text { testagem e aconselhamento. }\end{array}$ & $\begin{array}{l}\text { FERREIRA, } \\
\text { C. de O. et al. } \\
2019\end{array}$ & $\begin{array}{l}\text { Estudo analítico } \\
\text { observacional } \\
\text { transversal e } \\
\text { exploratório. }\end{array}$ & $\begin{array}{l}\text { Dados } \\
\text { secundários } \\
\text { de serviço de } \\
\text { saúde }\end{array}$ & $\begin{array}{l}\text { A prevalência de IST nesta população de } \\
\text { idosos foi } 25,32 \% \text {, com percentual mais } \\
\text { elevado entre os homens do que entre as } \\
\text { mulheres. A hepatite C foi a infecção } \\
\text { mais frequente, seguida da hepatite B, } \\
\text { sífilis e HIV. }\end{array}$ \\
\hline \begin{tabular}{lcc} 
Percepção & dos & Idosos \\
Relação a & Vida & Sexual e as \\
Infecções & \multicolumn{2}{c}{ Sexualmente } \\
Transmissíveis & na $\quad$ Terceira \\
Idade. & &
\end{tabular} & $\begin{array}{l}\text { THEIS, L. C.; } \\
\text { GOUVÊA, D. } \\
\text { L. } 2019\end{array}$ & $\begin{array}{l}\text { Estudo } \\
\text { qualitativo com } \\
\text { abordagem } \\
\text { descritiva. }\end{array}$ & $\begin{array}{l}\text { Entrevistas } \\
\text { semiestruturad } \\
\text { as com } \\
\text { perguntas } \\
\text { abertas }\end{array}$ & $\begin{array}{l}\text { Quando questionados sobre as infecções } \\
\text { sexualmente transmissíveis, todos os } \\
\text { idosos responderam que sabiam o que } \\
\text { eram, mas que não lembravam os nomes } \\
\text { das IST. As que foram lembradas por } \\
\text { eles foram: HIV, AIDS, sífilis e } \\
\text { gonorreia. }\end{array}$ \\
\hline 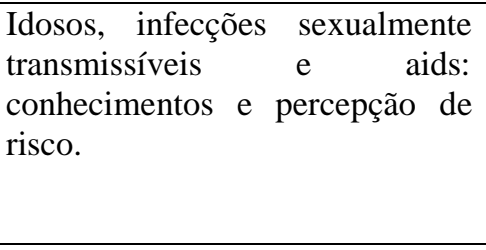 & $\begin{array}{l}\text { BRITO, N. } \\
\text { M. I. et al. } \\
2016\end{array}$ & $\begin{array}{l}\text { Estudo } \\
\text { descritivo de } \\
\text { natureza } \\
\text { quantitativa. }\end{array}$ & Formulário & $\begin{array}{l}\text { Em relação aos modos de prevenção, } \\
40 \% \text { dos idosos citaram o uso do } \\
\text { preservativo como principal método de } \\
\text { prevenção às infecções sexuais e } 20 \% \\
\text { não souberam informar sobre as medidas } \\
\text { preventivas. }\end{array}$ \\
\hline $\begin{array}{l}\text { Idosos e infecções sexualmente } \\
\text { transmissíveis: um desafio para a } \\
\text { prevenção. }\end{array}$ & $\begin{array}{l}\text { REIS, I.F. et } \\
\text { al. } 2020\end{array}$ & $\begin{array}{l}\text { Estudo } \\
\text { descritivo. }\end{array}$ & Entrevista & $\begin{array}{l}\text { Dentre os idosos } 77,1 \% \text { responderam } \\
\text { afirmativamente saber o que é uma } \\
\text { infecção sexualmente transmissível } \\
\text { (IST), enquanto } 22,8 \% \text { alegaram } \\
\text { desconhecimento. }\end{array}$ \\
\hline $\begin{array}{l}\text { Percepções sobre AIDS e } \\
\text { comportamento sexual em idosos } \\
\text { da cidade de Tubarão, Santa } \\
\text { Catarina. }\end{array}$ & $\begin{array}{l}\text { SAGGIORAT } \\
\text { O, A. K. S.; } \\
\text { TREVISOL } \\
\text { F. S. } 2015\end{array}$ & $\begin{array}{l}\text { Estudo } \\
\text { transversal. }\end{array}$ & $\begin{array}{l}\text { Entrevistas } \\
\text { semiestruturad } \\
\text { as }\end{array}$ & $\begin{array}{l}\text { Quanto ao conhecimento acerca de AIDS } \\
\text { e prevenção, a média de acerto foi de } \\
15 \pm 4 \text { de um total de } 25 \text { pontos. }\end{array}$ \\
\hline $\begin{array}{lr}\text { PERFIL DE } & \text { IDOSOS } \\
\text { PROCURAM O CENTRO DE } \\
\text { TESTAGEM } & \text { E } \\
\text { ACONSELHAMENTO } & \text { DE } \\
\text { IST/AIDS NO MUNICÍPIO DO } \\
\text { RECIFE }\end{array}$ & $\begin{array}{l}\text { JUNQUEIRA } \\
\text {, M. S. } 2019\end{array}$ & $\begin{array}{l}\text { Estudo } \\
\text { documental, } \\
\text { retrospectivo, } \\
\text { transversal, } \\
\text { quantitativo de } \\
\text { caráter } \\
\text { descritivo. } \\
\end{array}$ & $\begin{array}{l}\text { Análise de } \\
\text { prontuários }\end{array}$ & $\begin{array}{l}\text { O uso do preservativo ainda é uma } \\
\text { prática bastante ignorada nas relações } \\
\text { sexuais. Muitas questões colaboram para } \\
\text { a não utilização do preservativo durante } \\
\text { as relações sexuais, a fidelidade é dos } \\
\text { fatores expostos pela sociedade. }\end{array}$ \\
\hline
\end{tabular}




\begin{tabular}{|c|c|c|c|c|}
\hline $\begin{array}{lrr}\text { SEXUALIDADE } & \text { E } & \text { AIDS NA } \\
\text { TERCEIRA } & \text { IDADE: } \\
\text { ABORDAGEM } & \text { NA } \\
\text { CONSULTA MÉDICA } & \end{array}$ & $\begin{array}{l}\text { SANTOS, A. } \\
\text { M. } 2017\end{array}$ & $\begin{array}{l}\text { Estudo } \\
\text { transversal, de } \\
\text { natureza } \\
\text { quantitativa. }\end{array}$ & Questionário & $\begin{array}{l}\text { Dentre os médicos entrevistados, a } \\
\text { maioria relatou que os idosos os } \\
\text { questionam a respeito da sexualidade } \\
\text { durante a consulta médica. }\end{array}$ \\
\hline $\begin{array}{lcc}\text { Vulnerabilidade } & \text { de idosos a } \\
\text { infecções } & \text { sexualmente } \\
\text { transmissíveis } & \end{array}$ & $\begin{array}{l}\text { ANDRADE, } \\
\text { J. et al. } 2017\end{array}$ & $\begin{array}{l}\text { Estudo } \\
\text { transversal } \\
\text { analítico. }\end{array}$ & Entrevista & $\begin{array}{l}\text { A prevalência de IST foi } 3,4 \% \text { e entre as } \\
13 \text { ocorrências, havia } 10 \text { casos }(2,6 \%) \text { de } \\
\text { sífilis, dois }(0,5 \%) \text { de hepatite B e um } \\
(0,3 \%) \text { da infecção pelo HIV. A grande } \\
\text { maioria dos idosos }(82,2 \%) \text { não referiu } \\
\text { história de IST e } 20,4 \% \text { nunca havia } \\
\text { realizado exames sorológicos para } \\
\text { detecção destas doenças. }\end{array}$ \\
\hline $\begin{array}{l}\text { Práticas preventivas de idosos e } \\
\text { a vulnerabilidade ao HIV }\end{array}$ & $\begin{array}{l}\text { BEZERRA, } \\
\text { V. P. et al. } \\
2015\end{array}$ & $\begin{array}{l}\text { Estudo } \\
\text { exploratório } \\
\text { com abordagem } \\
\text { qualitativa. }\end{array}$ & Entrevista & $\begin{array}{l}\text { Predominância da faixa etária entre } 60 \text { e } \\
70 \text { anos }(64,8 \%) \text {, sexo feminino }(94,5 \%) \text {, } \\
\text { estado civil de viuvez, divorciadas e } \\
\text { solteiras }(54 \%) \text { e escolaridade de ensino } \\
\text { fundamental }(59 \%) \text {. A apreciação do } \\
\text { material empírico permitiu classificação } \\
\text { de três categorias: Práticas preventivas à } \\
\text { infecção pelo HIV; Facilidades e } \\
\text { Dificuldades no uso das práticas } \\
\text { preventivas e Pessoas vulneráveis ao } \\
\text { HIV. }\end{array}$ \\
\hline $\begin{array}{l}\text { Conhecimento e comportamento } \\
\text { de um grupo de idosos frente às } \\
\text { infecções } \\
\text { transmissíveis }\end{array}$ & $\begin{array}{l}\text { AMARAL, S. } \\
\text { V. A. } 2020\end{array}$ & $\begin{array}{l}\text { Estudo de } \\
\text { campo } \\
\text { exploratório- } \\
\text { descritivo, com } \\
\text { abordagem } \\
\text { quantitativa }\end{array}$ & Questionário & $\begin{array}{l}\text { Observa-se que com relação as } \\
\text { patologias que conheciam, } 64,4 \%(\mathrm{n}=38) \\
\text { referiu a doença HIV/AIDS e somente } \\
27,1 \% \text { (n=16,) afirmou conhecer todas as } \\
\text { doenças questionadas. Sobre a } \\
\text { importância do uso métodos de } \\
\text { prevenção durante a relação sexual, a } \\
\text { maioria respondeu corretamente sobre o } \\
\text { uso do preservativo para prevenir IST e } \\
\text { evitar gravidez. }\end{array}$ \\
\hline $\begin{array}{l}\text { Comportamento sexual de idosos } \\
\text { assistidos na estratégia saúde da } \\
\text { família }\end{array}$ & $\begin{array}{l}\text { LUZ, A. C. G. } \\
\text { et al. } 2015\end{array}$ & $\begin{array}{l}\text { Estudo } \\
\text { transversal, } \\
\text { exploratório e } \\
\text { quantitativo. }\end{array}$ & Entrevista & $\begin{array}{l}63,1 \% \text { dos idosos tinham vida sexual } \\
\text { ativa e } 20 \% \text { conversam sobre } \\
\text { sexualidade, mas apenas com amigos. } \\
12,3 \% \text { mantiveram desejo sexual total, } \\
57,7 \% \text { desejo parcial e } 30 \% \text { referiram } \\
\text { não ter mais nenhum desejo pelas } \\
\text { práticas sociais. Apenas } 10,8 \% \text { referiram } \\
\text { a utilização de método para prevenir IST, } \\
\text { e citaram exclusivamente o preservativo } \\
\text { masculino. }\end{array}$ \\
\hline
\end{tabular}




\begin{tabular}{|c|c|c|c|c|}
\hline $\begin{array}{l}\text { Sexuality through the eyes of the } \\
\text { elderly }\end{array}$ & $\begin{array}{l}\text { UCHÔA, Y. } \\
\text { da S. et al. } \\
2016\end{array}$ & $\begin{array}{l}\text { Estudo } \\
\text { quantitativo, } \\
\text { observacional } \\
\text { do tipo } \\
\text { transversal } \\
\text { analítico. }\end{array}$ & Entrevista & $\begin{array}{l}\text { As respostas sobre a investigação da } \\
\text { sexualidade durante a juventude } \\
\text { identificaram que, no início da vida } \\
\text { sexual dos idosos, houve diferença } \\
\text { significativa nos resultados, já que a } \\
\text { maioria dos idosos não se sentiam } \\
\text { preparados para iniciar a vida sexual e } \\
\text { relataram os amigos (outros) como } \\
\text { principal fonte de informações. }\end{array}$ \\
\hline \multirow[t]{2}{*}{$\begin{array}{l}\text { Vulnerability to HIV among } \\
\text { older men who have sex with } \\
\text { men users of dating apps in } \\
\text { Brazil }\end{array}$} & $\begin{array}{l}\text { QUEIROZ, } \\
\text { A. A. F. L. N. } \\
\text { et. al. } 2019\end{array}$ & $\begin{array}{l}\text { Estudo } \\
\text { transversal, de } \\
\text { base de dados }\end{array}$ & Questionário & $\begin{array}{l}\text { Em relação à presença de IST, } 22.1 \% \\
\text { reportaram pelo menos uma no ano } \\
\text { anterior ao questionário, com ênfase em } \\
\text { infecção por } \operatorname{HIV}(11.7 \%) \text { e } \quad \text { sífilis }\end{array}$ \\
\hline & & $\begin{array}{l}\text { populacional e } \\
\text { analítico. }\end{array}$ & & $\begin{array}{l}(10.2 \%) \text {. A forma mais comum de } \\
\text { prevenção foi o preservativo }(86.2 \%) \text { e } \\
47.3 \% \text { (195) dos indivíduos não tinham } \\
\text { testado para HIV recentemente (nos } 12 \\
\text { meses anteriores). }\end{array}$ \\
\hline $\begin{array}{l}\text { Aids em idosos: motivos que } \\
\text { levam ao diagnóstico tardio }\end{array}$ & $\begin{array}{l}\text { ALENCAR, } \\
\text { R. A. et. al. } \\
2016\end{array}$ & $\begin{array}{l}\text { Estudo } \\
\text { prospectivo, } \\
\text { com abordagem } \\
\text { qualitativa. }\end{array}$ & Questionário & $\begin{array}{l}\text { A solicitação da sorologia anti-HIV não é } \\
\text { rotina no serviço primário de saúde. A } \\
\text { solicitação da sorologia anti-HIV ocorreu } \\
\text { apenas aos idosos viúvos, com várias } \\
\text { parcerias sexuais ou usuários de drogas. }\end{array}$ \\
\hline $\begin{array}{l}\text { AIDS depois dos } 50 \text { anos: } \\
\text { incidência de } 2003 \text { a } 2013 \text { em } \\
\text { São José do Rio Preto, SP, e a } \\
\text { percepção dos idosos de uma } \\
\text { Unidade Básica de Saúde sobre a } \\
\text { doença }\end{array}$ & $\begin{array}{l}\text { CARVALHO, } \\
\text { N. Z. et. al. } \\
2017\end{array}$ & $\begin{array}{l}\text { Estudo } \\
\text { retrospectivo da } \\
\text { incidência de } \\
\text { AIDS a partir } \\
\text { dos casos } \\
\text { notificados no } \\
\text { sistema } \\
\text { eletrônico da } \\
\text { Secretaria de } \\
\text { Saúde do } \\
\text { Estado de São } \\
\text { Paulo entre } \\
2003 \text { e } 2013 \text {. }\end{array}$ & Questionário & $\begin{array}{l}\text { Responderam aos questionários } 34 \\
\text { homens e } 66 \text { mulheres entre } 50 \text { e } 88 \\
\text { anos. A maioria (59\%) referiu ter } \\
\text { parceiro fixo e negou a utilização de } \\
\text { preservativos (87\%). Após a atividade } \\
\text { educativa, apenas 5\% continuaram com } \\
\text { dúvidas e } 68 \% \text { demonstraram interesse } \\
\text { em obter informações sobre DTS na } \\
\text { UBS. }\end{array}$ \\
\hline $\begin{array}{l}\text { Conhecimento e atitudes sobre } \\
\text { sexualidade em pessoas idosas } \\
\text { com HIV }\end{array}$ & $\begin{array}{l}\text { AGUIAR, R. } \\
\text { B.; LEAL, M. } \\
\text { C. C.; } \\
\text { MARQUES, } \\
\text { A. P. O. } 2020\end{array}$ & $\begin{array}{l}\text { Estudo } \\
\text { descritivo, } \\
\text { quantitativo, de } \\
\text { corte } \\
\text { transversal. }\end{array}$ & Entrevista & $\begin{array}{l}\text { Na investigação acerca do conhecimento } \\
\text { e atitudes sobre sexualidade, os } \\
\text { participantes apresentaram um nível bom } \\
\text { de conhecimento (média de } 29,77 \text { ) e uma } \\
\text { atitude bastante positiva (média de } 14,85 \text { ) } \\
\text { sobre a sexualidade no envelhecimento. }\end{array}$ \\
\hline $\begin{array}{l}\text { PANORAMA } \\
\text { EPIDEMIOLÓGICO DA AIDS } \\
\text { EM IDOSOS }\end{array}$ & $\begin{array}{l}\text { SILVA, B. N. } \\
\text { et al. } 2018\end{array}$ & $\begin{array}{l}\text { Estudo } \\
\text { quantitativo, } \\
\text { descritivo, } \\
\text { retrospectivo, } \\
\text { com utilização } \\
\text { de dados } \\
\text { secundários. }\end{array}$ & $\begin{array}{l}\text { Utilização de } \\
\text { dados } \\
\text { secundários }\end{array}$ & $\begin{array}{l}\text { Os dados revelam que houve uma } \\
\text { tendência de crescimento linear no } \\
\text { número de registros de casos de AIDS } \\
\text { no Brasil nos últimos } 16 \text { anos, com } \\
\text { uma variação percentual } \\
\text { crescimento do número de casos de } \\
\text { aproximadamente } 185 \% \text { entre os anos de } \\
2000 \text { a } 2015 \text {. }\end{array}$ \\
\hline
\end{tabular}




\begin{tabular}{|c|c|c|c|c|}
\hline $\begin{array}{l}\text { HIV/aids em idosos: estigmas, } \\
\text { trabalho e formação em saúde }\end{array}$ & $\begin{array}{l}\text { CASSETTE, } \\
\text { J. B. et al. } \\
2016\end{array}$ & $\begin{array}{l}\text { Pesquisa } \\
\text { qualitativa, de } \\
\text { caráter } \\
\text { exploratório. }\end{array}$ & Entrevista. & $\begin{array}{l}\text { Com exceção de um dos profissionais de } \\
\text { saúde que relata manter um contato } \\
\text { bastante restrito e puramente técnico com } \\
\text { os pacientes, sem desenvolver com eles } \\
\text { maior vínculo, os demais percebem um } \\
\text { conjunto de prejuízos na vida social dos } \\
\text { idosos soropositivos, tais como } \\
\text { isolamento, afastamento das atividades } \\
\text { laborativas, religiosas e de lazer. }\end{array}$ \\
\hline $\begin{array}{lr}\text { SEXUALIDADE NO IDOSO: } \\
\text { PERCEPÇÃO } & \text { DE } \\
\text { PROFISSIONAIS } & \text { DA } \\
\text { GERIATRIA } & \text { E } \\
\text { GERONTOLOGIA } & \end{array}$ & $\begin{array}{l}\text { COSTA, } \\
\text { D.C.A. et al. } \\
2017\end{array}$ & $\begin{array}{l}\text { Estudo } \\
\text { quantitativo, } \\
\text { observacional, } \\
\text { do tipo } \\
\text { transversal } \\
\text { analítico. } \\
\end{array}$ & Questionário & $\begin{array}{l}\text { Todos os profissionais declaram saber } \\
\text { diferenciar sexo de sexualidade. A } \\
\text { maioria significativa teve de "nada" } \\
(45 \%) \text { a "pouco" }(30 \%) \text { embasamento } \\
\text { durante sua formação acadêmica } \\
\text { necessária para abordar o tema. E as }\end{array}$ \\
\hline & & & & $\begin{array}{l}\text { maiores dificuldades encontradas em } \\
\text { abordar o tema foram a "resistência dos } \\
\text { idosos" com } 50 \% \text { (10), "nenhum" } 30 \% \text { e } \\
\text { o "medo de ser invasivo" } 25 \% \text {. }\end{array}$ \\
\hline $\begin{array}{l}\text { Idosos cuidando de si após o } \\
\text { diagnóstico de síndrome da } \\
\text { imunodeficiência adquirida }\end{array}$ & $\begin{array}{l}\text { ARAUJO, G. } \\
\text { M. et al. } 2018\end{array}$ & $\begin{array}{l}\text { Pesquisa de } \\
\text { abordagem } \\
\text { qualitativa, } \\
\text { descritiva, } \\
\text { exploratória. }\end{array}$ & Questionário & $\begin{array}{l}\text { Em relação ao HIV/AIDS, antes de } \\
\text { serem diagnosticados, parte dos idosos } \\
\text { respondeu que não tinha nenhum } \\
\text { conhecimento. } \\
\text { O diálogo sobre sexualidade entre } \\
\text { profissionais e idosos parece ter ocorrido } \\
\text { somente após o diagnóstico da infecção } \\
\text { pelo HIV/AIDS. }\end{array}$ \\
\hline
\end{tabular}

\subsection{Desinformação por parte dos idosos e dos profissionais de saúde}

Constatou-se através da investigação epidemiológica direcionada à AIDS no idoso brasileiro feita por Silva et. al. (2018), que houve uma tendência de crescimento linear no número de registros da doença no Brasil nos últimos anos; com variação percentual de crescimento do número de casos aproximadamente $185 \%$ entre os anos 2000 e 2015 . Nesse cenário, no que tange à desinformação relacionada à precária educação sexual entre a população idosa, ressaltada pela ausência de políticas públicas voltadas à sexualidade nessa fase da vida, evidenciou-se o baixo conhecimento acerca da prevenção e transmissão do HIV, com informações superficiais e até mesmo distorcidas, como o pensamento de que a doença causaria a morte imediata, tal qual constatado nos estudos de Saggiorato; Trevisol (2015) e Araujo et al. (2018).

Ademais, é imprescindível salientar que a escassez de campanhas e atividades educativas sobre sexualidade, transmissão, prevenção e tratamento de IST, além de apoio social e psicológico, poderiam amenizar as consequências às pessoas que apresentam alguma 
IST e evitar maior contágio dessas infecções, tal como proposto por Nardelli et al. (2016) e Okuno et al. (2015). Nesse quesito, essa deficiente abordagem sobre sexualidade na terceira idade, mostrou que grande parte dos idosos não são capazes de distinguir sexo de sexualidade, deixando evidente o déficit na base da construção do conhecimento sobre esse assunto na senilidade, assim como demonstrado por Uchôa et al. (2016).

A questão da desinformação aliada ao desconhecimento dos idosos acerca de sexualidade, está bem evidenciada também no estudo de Nascimento et al. (2013), o qual reitera os resultados previamente encontrados, relatando o baixo nível de conhecimento sobre os conceitos, as formas de prevenção, de transmissão e vulnerabilidade em relação ao HIV/AIDS em idosos usuários do serviço público de saúde. Com relação ao escasso enfoque sobre sexualidade na terceira idade, no estudo de Laroque et al. (2011), revela que há grande dificuldade dos profissionais da saúde de falar sobre sexualidade nessa faixa etária em questão, pois a abordagem do conhecimento e do comportamento relacionado às DST/AIDS são, em geral, direcionados apenas a alguns grupos específicos, que excluem os idosos.

Diante desses fatores citados, tem-se que é necessária a própria conscientização da equipe em considerar a vida sexual do idoso como uma realidade, e, além disso, é de grande importância a orientação sobre esse assunto para essa população específica, objetivando uma maior inclusão dos idosos como público-alvo em políticas públicas sobre sexualidade e, assim, maior difusão de conhecimento e informação.

Constatou-se que o conhecimento sobre IST e suas formas de prevenção, contágio e tratamento é reduzido entre essa população. Os estudos de Reis et al. (2020) e Brito et al. (2016) apontaram concepções errôneas que os idosos possuem sobre as formas de transmissão de IST, em especial à transmissão relacionada ao HIV. No último estudo, reitera-se a ideia de que idosos se consideram imunes à infecção por possuírem um único parceiro, e que relatam o uso de preservativos apenas quando não há confiança em tal parceiro.

O fato de os idosos possuírem concepções errôneas foi também avaliado por Bastos et al. (2018). Apesar de ter constatado em sua pesquisa que 96,4\% dos entrevistados tinham conhecimento da AIDS; 67,3\% dos idosos afirmaram que desconheciam a sífilis quando questionados sobre a doença, e 70,9\% não possuíam informações sobre sua forma de transmissão. Em relação aos meios de transmissão do HIV; 78,1\% acreditavam que poderia ser transmitido através da picada de mosquito, $40 \%$ pelo uso do mesmo copo, e $38,1 \%$ dos idosos entrevistados acreditava que seria viável por meio do beijo na boca. Entre os entrevistados, 30,9\% referiram ter vida sexual ativa. No entanto, no que se refere ao uso de preservativos; 90,9\% afirmaram que não usavam e nunca haviam usado preservativos durante 
a relação sexual. Os dados do estudo demonstraram que aqueles que os usam têm média de conhecimento maior.

\subsection{Aspectos culturais}

Segundo os autores Brito et al. (2016), Amaral et al. (2020) e Andrade et al. (2017), a maioria dos idosos acreditam não serem vulneráveis às infecções $(81,4 \%)$, já que $76,4 \%$ dos entrevistados responderam não ter nenhuma possibilidade de adquirir infecções sexualmente transmissíveis por terem parceiros fixos ou por ausência de atividade sexual. Nesse sentido, os idosos entendem que a vulnerabilidade se faz presente, em sua maioria, em jovens, em homossexuais e em pessoas sem parceiro fixo, mas pouca ênfase é dada à vulnerabilidade sofrida nessa faixa etária mais avançada, consoante a Bezerra et al. (2015). Dessa forma, de acordo com o estudo de Amaral et al. (2020), 40,7\% dos idosos não se sentem à vontade para falar sobre sexualidade, seja por acreditarem que idosos não têm relação sexual ou até mesmo por sentirem vergonha.

Sustentando essa lógica, os estudos de Moreira et al. (2015) e de Silva et al. (2017) trazem à tona que a falta de uma maior busca por conhecimento em educação sexual para a população idosa influencia no desenvolvimento de atitudes negativas frente à sexualidade, o que os torna ainda mais suscetíveis. O primeiro estudo ainda pontua como mais um fator preponderante à persistência desse cenário os hiatos nas políticas públicas na Estratégia de Saúde da Família na atenção primária, pois estas deveriam cumprir o papel de conscientizar e orientar, a partir de uma relação mais estreita, os idosos a respeito de sua vulnerabilidade. Logo, estudos sugerem que a expressão sexual deve permanecer por toda a vida e se todas essas medidas não forem tomadas, a qualidade de vida dos idosos continuará sendo influenciada negativamente em função de tabus e ausência de diálogo, como pontua Carmenates e Bujardón (2010), Lindau et al. (2011) e Hoekstra et al. (2012).

Paralelamente, os profissionais de saúde compõem um fator preponderante para a manutenção do aumento na transmissibilidade de doenças infecciosas em idosos no Brasil. Isso porque, além de tais profissionais não terem uma formação acadêmica que efetivamente abordasse a questão da sexualidade na terceira idade, a negligência profissional é comum nas redes de atenção, visto que, até mesmo aqueles que possuem o conhecimento adequado sobre assunto possuem atitudes conservadoras, por razões culturais, que dificultam o diálogo, de acordo com os estudos de Alencar et al. (2016), Costa et al. (2017), Araujo et al. (2018). A 
negligência profissional relacionada à questão cultural foi também evidenciada nos estudos de Evangelista et al. (2017), Cassette et al. (2016) e Cherpak; Santos (2016).

Outro fator relacionado ao serviço de saúde prestado ao idoso que sustenta esse cenário é relativo à sorologia para HIV, a qual possui uma baixa taxa de solicitação por ser requisitada apenas quando o paciente apresenta queixas ou clara instalação da infecção, segundo as perspectivas de Junqueira et al. (2019), Santos et al. (2017) e Alencar et al. (2016). Dessa forma, segundo Luz et al. (2015), os idosos atribuem a maior parte de informação adquirida sobre as IST aos meios de comunicação e não aos profissionais de saúde.

A perspectiva de Venturini et al. (2018) e Taylor e Gosney (2011), que corrobora com a ideia anterior, mostra que os profissionais de saúde apresentam dificuldade em abordar o tema da sexualidade com idosos, por constrangimento ou por ter dificuldade de separar a realidade do paciente de suas crenças pessoais, o que escancara as falhas na formação acadêmica e profissional. Essa ausência de diálogo impede, por exemplo, a realizações de testes rápidos de IST que trariam um possível diagnóstico precoce e, por isso, um mais fácil tratamento, não afetando de maneira considerável a qualidade de vida dos idosos infectados. Ainda, assim, os profissionais que trazem essa realidade ao atendimento tendem a explorarem somente tratamento a partir de queixas e não souberam, na pesquisa feita por Cunha et al. (2015), traçar estratégias de prevenção e promoção da saúde.

\subsection{Práticas sexuais inseguras}

A príncipio, no que diz respeito às práticas sexuais inseguras, investigou-se e constatou-se que o uso de preservativos é um hábito comumente negligenciado pela população idosa, assim como evidenciado pelo estudo de Queiroz et al. (2019), no qual a maioria dos idosos (59\%) referiu ter parceiro fixo e negou a utilização de preservativos (87\%). Em concordância com esse fato, os estudos de Theis; Gouvêa (2019) e Ferreira et al. (2019) apontaram que o entendimento errôneo de que preservativos contribuem apenas com a finalidade de contracepção cauciona a problemática em relação às práticas sexuais riscosas. Verificou-se ainda neste lamiré, demasiada desinformação, por parte da população idosa, a respeito dos riscos de transmissão de infecções sexualmente transmissíveis (IST).

Além disso, segundo os estudos de Reis et al. (2020), o não uso de preservativos tem também relação direta com a dificuldade de negociação entre os parceiros, a qual é 
obstaculizada com a resistência e não aceitação do uso por parte de um ou de ambos. Nessa perspectiva, notou-se que não há negociação do uso de preservativos, sobretudo, quando existe confiança no parceiro, por este ser o único. Diante disso, é reforçado o ideal proposto por Amaral (2020), que afirma que o idoso tem reduzida percepção em relação à sua vulnerabilidade, o que reflete na banalização da prática sexual insegura por essa população.

Em contraste, o estudo de Queiroz, et. al. (2019), conduzido com participantes que utilizam aplicativos de namoro, homens que fazem sexo com homens (HSH) de todo o Brasil, sendo a maioria solteiros, revelou o preservativo como a forma mais comum de prevenção $(86,2 \%)$. No entanto, os resultados mostraram uma alta prevalência de HIV entre HSH com idade de 50 anos ou mais $(11,7 \%)$, que são usuários de aplicativos de relacionamento no Brasil, especialmente quando comparados com a população geral $(0,39 \%)$.

Sustentando a problemática de práticas sexuais inseguras, os estudos de Cerqueira; Rodrigues (2016), por outro lado, denotaram que há resistência em relação ao uso de preservativos também pelo juízo de que a relação sexual não se desenvolve com prazer equivalente à desprotegida. Esse contexto torna-se ainda mais preocupante, visto que segundo os ideais desses autores, algumas idosas partícipes da amostra revelaram que os companheiros, mesmo sabendo da condição sorológica positiva para AIDS, recusam-se a usar preservativos.

Achados semelhantes foram encontrados nos estudos de Paulino, et al. (2014), os quais quantificaram que dentre os idosos com vida sexual ativa, 64\% não utilizaram preservativo na última relação, apesar de seu uso ser apontado como conduta preventiva de infecção pelo HIV. Ainda nesse quesito, houve grande disparidade entre os homens e mulheres: mais de $90 \%$ dos homens não usaram preservativo contra apenas duas das 93 mulheres não usaram. A justificativa das idosas para o não uso de preservativo é a possibilidade de ruptura no momento do ato ou empecilho para o prazer, o que contribui para a perpetuação da prática sexual insegura por idosos.

\section{CONCLUSÃO}

Mediante todos os trabalhos analisados, a prática sexual insegura encontra-se entre os principais fatores que influenciam na transmissão de IST. Foi demonstrado que a justificativa dada pelos idosos é a de possuírem parceiros fixos, devido à ideia de que o único objetivo dos preservativos é a contracepção. Outro fator que está intrinsecamente adistrito à transmissão de 
IST entre os idosos no país são os aspectos culturais. Nessa linha, falsas crenças, como na impossibilidade de uma pessoa mais velha se infectar sexualmente, mascaram a vulnerabilidade sofrida por essa população. Além disso, profissionais de saúde relataram dificuldade de questionar sobre o tema com pacientes idosos, por sentirem vergonha, escancarando a insuficiente formação acadêmica em relação ao cenário exposto.

Por postimeiro, a desinformação por parte dos idosos e dos profissionais de saúde se apresentam como um fator associado à problemática. Há uma ausência de políticas públicas suficientes que abordem o tema na atenção primária, e de campanhas de ações educativas acerca da importância do conhecimento sobre sexualidade e sobre a transmissão, prevenção e tratamento de IST no Brasil. Diante disso, faz-se necessário o desenvolvimento de pesquisas e de políticas públicas que englobem o idoso nas campanhas de prevenção de IST, visto que, como retratado neste estudo, a prevenção é pouco abordada, levando então a infecções que poderiam ser evitadas e ao diagnóstico muitas vezes tardio dessas infecções. Dessa forma, ações de promoção à saúde visando o bem-estar do idoso e não apenas o foco na doença também se fazem necessárias.

\section{REFERÊNCIAS}

AGUIAR, Rosaline Bezerra; LEAL, Márcia Carréra Campos; MARQUES, Ana Paula de Oliveira. Conhecimento e atitudes sobre sexualidade em pessoas idosas com HIV. Ciênc. saúde coletiva, Rio de Janeiro, v. 25, n. 6, p. 2051-2062, 2020. Disponível em: <https://doi.org/10.1590/1413-81232020256.18432018>. Acesso em: 25 set. 2020.

ALENCAR, R. A.; CIOSAK, S. I. Aids em idosos: motivos que levam ao diagnóstico tardio. Revista Brasileira de Enfermagem, v. 69, n. 6, p. 1076-81, 2016. Disponível em: <https://doi.org/10.1590/0034-7167-2016-0370>. Acesso em: 28 ago. 2020.

AMARAL, S. V. A. et al. Conhecimento e comportamento de um grupo de idosos frente às infecções sexualmente transmissíveis. Revista eletrônica Acervo Saúde, v. 12, n. 9, p. 1-12, 2020. Disponível em: <https://doi.org/10.25248/reas.e3891.2020>. Acesso em: 25 set. 2020.

ANDRADE, J. et al. Vulnerabilidade de idosos a infecções sexualmente transmissíveis. Acta Paul Enferm., v. 30, n. 1, p. 8-15, 2017. Disponível em: <https://doi.org/10.1590/19820194201700003>. Acesso em: 27 set. 2020. 
ANDRADE, P.B.S.; BENITO, L.A.O. Perfil da sexualidade de pessoas idosas portadoras de SIDA/AIDS atendidas em um serviço de saúde do Distrito Federal. Universitas: Ciências da Saúde, v. 14, n. 2, p. 105-113, 2016. Disponível em:

<https://doi.org/10.5102/ucs.v14i2.3812>. Acesso em: 15 set. 2020.

ARAUJO, G. M. et al. Idosos cuidando de si após o diagnóstico de síndrome da imunodeficiência adquirida. Rev. Bras. Enferm., v. 71, supl. 2, p. 793-800, 2018. Disponível em: <https://doi.org/10.1590/0034-7167-2017-0248>. Acesso em: 15 set. 2020.

BASTOS, L. M. et al. Avaliação do nível de conhecimento em relação à Aids e sífilis por idosos do interior cearense, Brasil. Ciênc. saúde coletiva, v. 23, n. 8, p. 2495-2502, 2018. Disponível em: <https://doi.org/10.1590/1413-81232018238.10072016>. Acesso em: 25 set. 2020. 
BEZERRA, V. P. et al. Práticas preventivas de idosos e a vulnerabilidade ao HIV. Revista Gaucha de Enfermagem, v. 36, n. 4, 70-76, 2015. Disponível em:

<https://doi.org/10.1590/1983-1447.2015.04.44787>. Acesso em: 27 set. 2020.

BRASIL. Ministério de Saúde. Portaria n. 2528/GM, de 19 de outubro de 2006. Aprova a Política Nacional de Saúde da Pessoa Idosa. Brasília; 2006. Disponível em:

<http://bvsms.saude.gov.br/bvs/saudelegis/gm/2006/prt2528_19_10_2006.html>. Acesso em: 27 set. 2020.

BRITO, N. M. I., et. al. Idosos, infecções sexualmente transmissíveis e aids: conhecimentos e percepção de risco. Abcs Health Sciences, v. 41, n. 3, p.140-145, 2016. Disponível em: <HYPE//dx.doi.org/10.7322/abcshs.v41i3.902>. Acesso em: 27 set. 2020.

CARMENATES, C.O.; MENDONZA, A.B. Estrategia educativa para lograr una sexualidad saludable en el adulto mayor. Ver Hum Med, v. 10, n. 2, p. 1-22, 2010. Disponível em: $<$ http://scielo.sld.cu/scielo.php?script=sci_arttext\&pid=S1727-

$81202010000200006 \& \operatorname{lng}=$ es\&nrm=iso>. Acesso em: 25 set. 2020.

CARVALHO, N. Z., et al. AIDS depois dos 50 anos: incidência de 2003 a 2013 em São José do Rio Preto, SP, e a percepção dos idosos de uma Unidade Básica de Saúde sobre a doença. DST - Jornal Brasileiro de Doenças Sexualmente Transmissíveis, v. 29, n. 3, p. 85-90, 2017. Disponível em: <10.5533/DST-2177-8264-201729303>. Acesso em: 28 ago. 2020.

CASSETTE, J. B. et al. HIV/aids em idosos: estigmas, trabalho e formação em saúde. Rev. bras. geriatr. gerontol., Rio de Janeiro. v. 19, n. 5, p. 733-744, 2016. Disponível em: <https://doi.org/10.1590/1809-98232016019.150123>. Acesso em: 25 set. 2020.

CERQUEIRA, M. B, R.; RODRIGUES, R. N. Fatores associados à vulnerabilidade de idosos vivendo com HIV/AIDS em Belo Horizonte (MG), Brasil. Ciên. saúde coletiva, v. 21, n. 11, p. 3331-3338, 2016. Disponível em: <https://doi.org/10.1590/1413-

812320152111.14472015>. Acesso em: 28 set. 2020.

CHERPAK, G.L.; SANTOS, F.C. Avaliação da abordagem médica da sexualidade em idosos com dor crônica. Einstein (São Paulo), v. 14, n. 2, p. 178-184, 2016. Disponível em: <https://doi.org/10.1590/S1679-45082016AO3556>. Acesso em: 15 set. 2020. 
COSTA, D. C. A. et al. Sexualidade no idoso: percepção de profissionais da geriatria e gerontologia. Universitas: Ciências Da Saúde, Brasília, v. 15, n. 2, p. 75-80, 2017. Disponível em: <HYPE://doi.org/10.5102/ucs.v15i2.3997>. Acesso em: 28 set. 2020.

CUNHA, L.M. et al. Grandma and Grandpa also love: sexuality in the elderly. Rev Min Enferm., v. 19, n. 4, p. 894-900, 2015. Disponível em:

<http://www.dx.doi.org/10.5935/1415-2762.20150069>. Acesso em: 28 set. 2020.

EVANGELISTA, A.R. et al. Sexuality in old age: knowledge/attitude of nurses of Family Health Strategy. Rev. esc. enferm. USP, v. 53, e03482, 2019. Disponível em: <https://doi.org/10.1590/S1980-220X2018018103482>. Acesso em: 25 set. 2020.

FERREIRA, C. de O., et al. Vulnerabilidade a infecções sexualmente transmissíveis em idosos usuários de um centro de testagem e aconselhamento. Arq. Cienc. Saúde UNIPAR, Umuarama, v. 23, n. 3, p.171-180, 2019. Disponível em:

<https://doi.org/10.25110/arqsaude.v23i3.2019.6757>. Acesso em: 30 set. 2020.

HOEKSTRA, T. et al. Perceived sexual difficulties and associated factors in patients with heart failure. Am Heart, v. 163, n. 2, p. 246-251, 2012. Disponível em: <http://dx.doi.org/10.1016/j.ahj.2011.10.011>. Acesso em: 30 set. 2020.

JUNQUEIRA, M. S. et al. Perfil de idosos que procuram o centro de testagem e aconselhamento de IST/AIDS no município do Recife. Revista Educação, Meio Ambiente e Saúde, v. 9, n.3, p. 126-136, 2019. Disponível em:

LAROQUE, M. F. et al. Sexualidade do idoso: Comportamento para prevenção de DST/AIDS. Rev. Gaúcha Enferm., v. 32, n. 4, p. 774-80, 2011. Disponível em: <https://doi.org/10.1590/S1983-14472011000400019>. Acesso em: 30 set. 2020.

LINDAU, S.T. et al. Communication about Sexuality and Intimacy in Couples Affected by Lung Cancer and their Clinical Care Providers. Psychooncology, v. 20, n. 2, p. 179-185, 2011. Disponível em: <10.1002/pon.1787>. Acesso em: 30 set. 2020. 
LUZ, A. C. G. et al. Comportamento sexual de idosos assistidos na estratégia saúde da família. Revista de pesquisa Cuidado é fundamental, v. 7, n. 2, p. 2229-2240, 2015. Disponível em: <https://www.redalyc.org/articulo.oa?id=505750946004>. Acesso em: 28 ago. 2020.

MIRANDA, G. M. D.; MENDES, A. C. G.; SILVA, A. L. A. O envelhecimento populacional brasileiro: desafios e consequências sociais atuais e futuras. Revista Brasileira de Geriatria e Gerontologia, v. 19, n.3, p. 507-519, 2016. Disponível em: <https://doi.org/10.1590/180998232016019.150140>. Acesso em: 25 set. 2020.

MOREIRA W.C. et al. Sexualidade e prevenção de IST e HIV/aids entre idosos usuários da estratégia saúde da família. Rev. Pre. Infec e Saúde, v. 1, n. 3, p. 76-82, 2015. Disponível em: < https://doi.org/10.26694/repis.v1i3.3943>. Acesso em: 27 set. 2020.

NARDELLI, G.G. et al. Conhecimento sobre síndrome da imunodeficiência humana de idosos de uma unidade de atenção ao idoso. Rev. Gaúcha Enferm., v. 37, e2016-0039, 2016. Disponível em: <https://doi.org/10.1590/1983-1447.2016.esp.2016-0039>. Acesso em: 30 set. 2020.

NASCIMENTO, R. G. et al. Nível de conhecimento de idosos comunitários em relação ao HIV/Aids: estudo exploratório na rede básica de saúde de Belém, Pará, Brasil. Revista Brasileira De Ciências Do Envelhecimento Humano, v. 10, n. 1, p. 113-122, 2013. Disponível em: < https://doi.org/10.5335/rbceh.2012.3018>. Acesso em: 25 set. 2020.

OKUNO, M.F.P. et al. Quality of life, socioeconomic profile, knowledge and attitude toward sexuality from the perspectives of individuals living with Human Immunodeficiency Virus. Rev. Latino-Am. Enfermagem, v. 23, n. 2, p. 192-199, 2015. Disponível em: <https://doi.org/10.1590/0104-1169.3424.2542>. Acesso em: 30 set. 2020.

PAULINO, M.C.de F. Análise dos comportamentos sexuais de idosos cadastrados em uma Estratégia Saúde da Família. Revista Kairós Gerontologia, v. 17, n. 4, p. 49-61, 2014. Disponível em: < https://revistas.pucsp.br/index.php/kairos/article/download/23396/16829>. Acesso em: 28 set. 2020.

QUEIROZ, A. A. F. L. N., et al. Vulnerability to HIV among older men who have sex with men users of dating apps in Brazil. The Brazilian Journal of Infectious Diseases, v. 23, n. 5, 
p. 298- 306, 2019. Disponível em: <https://doi.org/10.1016/j.bjid.2019.07.005>. Acesso em: 28 set. 2020.

REIS, I. F., et al. Idosos e infecções sexualmente transmissíveis: um desafio para a prevenção. Brazilian Journal of health Review, v. 3, n. 2, p. 1663- 1675, 2020. Disponível em: < HYPE://doi.org/10.34119/bjhrv3n2-028>. Acesso em: 15 set. 2020.

SAGGIORATO, A. K. S.; TREVISOL, F. S. Percepções sobre AIDS e comportamento sexual em idosos da cidade de tubarão, Santa Catarina. Jornal Brasileiro de Doenças Sexualmente Transmissíveis, v. 27, n. 1-2, p. 29-34, 2015. Disponível em:

< HYPE//www.dst.uff.br/revista27-1-2-2015/DST_v27n1-2_29-34_IN.pdf>. Acesso em: 30 set. 2020.

SANTOS, M. A. et al. Sexualidade e AIDS na terceira idade: Abordagem na consulta médica. Rev. Aten. Saúde, v. 15, n. 51, p. 18-22, 2017. Disponível em: < https://doi.org/10.13037/ras.vol15n51.4152>. Acesso em: 28 set. 2020.

SILVA, B. N. et al. PANORAMA EPIDEMIOLÓGICO DA AIDS EM IDOSOS. Hygeia Revista Brasileira de Geografia Médica e da Saúde, v. 14, n. 29, p. 80 - 88, 2018. Disponível em: < HYps://doi.org/10.14393/Hygeia142907>. Acesso em: 25 set. 2020.

SILVA, J. D. B. et al. Vulnerabilidade às infecções sexualmente transmissíveis/AIDS em idosos. Revista Uningá, v. 53, n. 1, p. 19-24, 2017. Disponível em: <http://revista.uninga.br/index.php/uninga/article/view/1418>. Acesso em: 28 ago. 2020.

SOUSA, M. C. et al. O envelhecimento da população: aspectos do Brasil e do mundo, sob o olhar da literatura. Braz. J. of Develop., v. 6, n. 61871-61877, p. 1-7, 2020. Disponível em: < https://doi.org/10.34117/bjdv6n8-564>. Acesso em: 30 set. 2020.

TAYLOR, A.; GOSNEY, M.A. Sexuality in older age: essential considerations for healthcare professionals. Age Ageing, v. 40, n. 5, p. 538-543, 2011. Disponível em: < 10.1093/ageing/afr049>. Acesso em: 27 set. 2020. 
THEIS, L. C.; GOUVÊA, D. L. Percepção dos Idosos em Relação a Vida Sexual e as Infecções Sexualmente Transmissíveis na Terceira Idade. Revista Brasileira de Ciências da Saúde, v.23, n. 2, p. 197-204, 2019. Disponível em: <https://doi.org/10.22478/ufpb.23176032.2019v23n2.36926>. Acesso em: 15 set. 2020.

UCHÔA, Y. S. et al. A sexualidade sob o olhar da pessoa idosa. Revista brasileira de Geriatria e Gerontologia, v.19, n. 6, p. 939-949, 2016. Disponível em: <https://doi.org/10.1590/1981-22562016019.150189>. Acesso em: 28 ago. 2020.

VENTURINI, L. et al. Atuação da equipe de enfermagem frente à sexualidade de idosas institucionalizadas. Rev. esc. enferm. USP, v. 52, e03302, 2018. Disponível em: <https://doi.org/10.1590/S1980-220X2017017903302>. Acesso em: 25 set. 2020. 